\title{
La ventilación mecánica invasiva en los adultos mayores
}

\author{
Vanesa Romina Ruiz ${ }^{\oplus}$ y María Florencia Grande Ratti ${ }^{\oplus}$
}

Ruiz VR, Grande-Ratti MF, Martínez B, Midley Rt A, Sylvestre V, Mayer GF. In-hospital mortality associated factors in elderly patients with invasive mechanical ventilation in the emergency department. Enferm Intensiva. 2021 Jul-Sep;32(3):145-52. doi: 10.1016/j.enfi.2020.08.002.

\section{COMENTARIO}

Según nuestro estudio de cohorte retrospectiva, la tasa de ventilación mecánica invasiva (VMI) de pacientes mayores de 65 años en la Central de Emergencias de Adultos (CEA) fue de $0,48 \%$ (IC 95\% 0,43-0,54) entre junio de 2016 y mayo de 2018 . Hubo un total de 285 intubados en Guardia de un total de 58403 consultas consecutivas correspondientes a las áreas de atención A (Cuidados Críticos), B (Cuidados Intermedios) y C (Consultorios de moderada complejidad), restringiendo el denominador a los afiliados de la prepaga institucional con ese rango etario.

En nuestra CEA, la intubación orotraqueal (IOT) se realiza en el área A siguiendo la secuencia rápida de intubación. Pese a la baja prevalencia de este procedimiento en adultos mayores $(<1 \%)$, es un dato que resultó de gran relevancia local, clínica y gerencial. En el contexto del envejecimiento poblacional debido al cambio demográfico y al aumento de expectativa de vida, la demanda de este grupo etario a los servicios de salud, incluidos los servicios de emergencias, va en ascenso ${ }^{1}$. Es interesante destacar que en junio de 2018, los mayores de 65 años representaban el 33\% del padrón de afiliados al Plan de Salud. Sin embargo, durante el período de estudio, realizaron más de la mitad de las consultas a la CEA, el 67\% había consultado en el año previo y más de un tercio de ellos había requerido internación.

Cada vez con más frecuencia, estos pacientes, particularmente frágiles y vulnerables, pueden requerir IOT en el contexto de una enfermedad crítica, que resulta entonces un procedimiento de rápida decisión y situación algo dificultosa ${ }^{2}$. Si bien la VMI puede permitir la supervivencia en el período agudo, la mortalidad intrahospitalaria o extrahospitalaria, o ambas, a corto-mediano plazo es

Recibido: $2 / 8 / 21$

Aceptado: $16 / 8 / 21$

Sección de Rehabilitación y Cuidados Respiratorios del Paciente Crítico (V.R.R.). Área de Investigación en Medicina Interna (M.F.G.R.), Hospital Italiano de Buenos Aires. Maestría en Investigación Clínica (M.F.G.R.), Instituto Universitario Hospital Italiano de Buenos Aires. Buenos Aires, Argentina.

Correspondencia: maria.grande@hospitalitaliano.org.ar elevada en esta población ${ }^{3}$. Incluso en los sobrevivientes, la VMI puede resultar prolongada, al igual que la estadía hospitalaria, con baja calidad de vida al alta y consecuentes costos elevados en salud ${ }^{4}$. Es por esto que algunos autores sugieren establecer cuidados limitados para evitar futilidad terapéutica. Sin embargo, los argumentos que se usan para la adecuación terapéutica están basados en la edad, la calidad de vida al alta o la probabilidad de supervivencia, siendo las últimas dos extremadamente difíciles de predecir en urgencias ${ }^{5}$. Asimismo, la mayoría de los estudios sobre factores asociados a mortalidad en adultos mayores fueron realizados en países desarrollados o en pacientes internados en unidades cerradas ${ }^{6}$.

Ante la inexistencia de modelos predictivos locales en el ámbito de emergencias para dicha decisión, el propósito de este trabajo fue identificar variables incluidas en la valoración geriátrica integral y otras variables clínicas al ingreso que se asocian a mortalidad intrahospitalaria después del requerimiento de IOT de urgencia. Más de la mitad de nuestros pacientes ancianos fallecieron durante su hospitalización. De hecho, nuestro hallazgo del 55\% superó la predicha por el puntaje (score) de APACHE II (40\%) -acrónimo de Acute Physiology And Chronic Health Evaluation-y de SOFA (33\%) -del inglés Sequential Organ Failure Assessment-. Los factores asociados a mortalidad tras el ajuste por edad y sexo resultaron: accidente cerebrovascular (OR 2,13; IC 95\%1,21-3,76), insuficiencia renal crónica (OR 4,38; IC 95\%1,91-10,04), índice de Charlson (OR 1,19; IC 95\%1,02-1,38), APACHE II (OR 1,07; IC 95\%1,02-1,12) y SOFA (OR 1,14; IC 95\%1,03-1,27).

La relevancia clínica de esta información radica en las implicaciones prácticas para la toma de decisiones compartidas en salud ${ }^{7,8}$. Para los adultos mayores existen desafíos y consideraciones específicas, como la presencia de comorbilidades, la capacidad funcional previa y la calidad de vida posterior al alta hospitalaria, además de las preferencias de los pacientes y familiares (p. ej., directivas anticipadas $)^{9}$. Las decisiones sobre el alcance de los esfuerzos de reanimación (o en este caso requerimiento de IOT en contexto agudo) deberían incorporar 
estas evaluaciones o variables. Sin embargo, muchas veces esta información no se encuentra disponible, no es factible su evaluación por la gravedad del cuadro, o la combinación de estos datos no aporta una estratificación pronóstica para la toma de decisión compartida de forma sencilla y rápida, como requiere un ámbito como la urgencia. Probablemente, esta información pueda ser el puntapié inicial para, en el futuro, poder contar con la generación de una herramienta predictiva de mal pronóstico. Asimismo se necesita la validación de puntajes de gravedad al ingreso específicos para los servicios de emergencia.

La principal fortaleza, a pesar de ser unicéntrico, radica en el volumen de pacientes de esta complejidad y en la factibilidad de recolectar la información relacionada con los datos clínicos y los registros personales de salud en la historia clínica electrónica como factor clave para la viabilidad.
Esta fue la fuente de recolección de datos retrospectivos necesarios para el proyecto.

A su vez, representó un esfuerzo y trabajo colaborativo en equipo interdisciplinario, con integrantes de la Sección de Rehabilitación y Cuidados Respiratorios del Paciente Crítico del Servicio de Kinesiología, del Área de Investigación en Medicina Interna de Clínica Médica, y de la Central de Emergencias de Adultos, lo que resultó una experiencia enriquecedora. Cabe destacar que, si bien esta problemática viene siendo abordada desde hace varios años en términos de frecuencia y costos en salud, resultó extremadamente necesaria y fue foco durante la pandemia COVID-19 sin precedentes, debido a los escenarios de sobrecupo en urgencias, contexto en el que el comité de crisis debía poder dar respuesta previsible a la demanda de recursos en las Unidades de Cuidados Críticos ${ }^{10}$.

Conflictos de interés: los autores declaran no tener conflictos de interés.

\section{REFERENCIAS}

1. Aminzadeh F, Dalziel WB. Older adults in the emergency department: a systematic review of patterns of use, adverse outcomes, and effectiveness of interventions. Ann Emerg Med. 2002;39(3):238-247. doi: 10.1067/ mem.2002.121523.

2. Lewis ET, Dent E, Alkhouri H, et al. Which frailty scale for patients admitted via Emergency Department? A cohort study. Arch Gerontol Geriatr. 2019;80:104-114. doi: 10.1016/j.archger.2018.11.002.

3. Cardona M, O'Sullivan M, Lewis ET, et al. Prospective validation of a checklist to predict short-term death in older patients after emergency department admission in Australia and Ireland. Acad Emerg Med. 2019;26(6):610620. doi: 10.1111/acem. 13664 .

4. Ouchi K, Jambaulikar GD, Hohmann S, et al. Prognosis after emergency department intubation to inform shared decision-making. J Am Geriatr Soc. 2018;66(7):1377-1381. doi: 10.1111/jgs. 15361 .

5. Carpenter CR, Shelton E, Fowler S, et al. Risk factors and screening instruments to predict adverse outcomes for undifferentiated older emergency department patients: a systematic review and meta-analysis. Acad Emerg Med. 2015;22(1):1-21. doi: 10.1111/ acem. 12569.

6. Giannasi SE, Venuti MS, Midley AD, et al. Factores de riesgo de mortalidad de los pacientes ancianos en cuidados intensivos sin limitación del esfuerzo de tratamiento. Med Intensiva. 2018;42(8):482-489. doi: 10.1016/j. medin.2017.10.014.

7. Backman WD, Levine SA, Wenger NK, et al. Shared decision-making for older adults with cardiovascular disease. Clin Cardiol. 2020;43(2):196-204. doi: 10.1002/clc.23267.

8. Truglio-Londrigan M, Slyer JT, Singleton JK, Worral P. A qualitative systematic review of internal and external influences on shared decision-making in all health care settings. JBI Libr Syst Rev. 2012;10(58):4633-4646. doi: 10.11124/jbisrir-2012-432.

9. Vargas N, Tibullo L, Landi E, et al. Caring for critically ill oldest old patients: a clinical review. Aging Clin Exp Res. 2017;29(5):833845. doi: 10.1007/s40520-016-0638-y.

10. Raheja H, Chukwuka N, Agarwal C, et al. Should COVID-19 patients $>75$ years be ventilated? An outcome study. QJM. 2021;114(3):182-189. doi: 10.1093/qjmed/ hcab029. 